\title{
New Applications for Affinity Grids in Preparing EM Specimens
}

\author{
Deborah F. Kelly, ${ }^{*}$ Danijela Dukovski ${ }^{*, * *}$ and Thomas Walz ${ }^{* * *}$ \\ * Department of Cell Biology and ** Howard Hughes Medical Institute, \\ Harvard Medical School, 240 Longwood Avenue, Boston, MA
}

We have previously developed the monolayer purification technique to prepare specimens for singleparticle electron microscopy (EM) without prior purification [1]. In this technique, a monolayer comprised of lipids with functionalized Nickel-nitrilotriacetic acid (Ni-NTA) head groups is formed over a solution or cell extract containing His-tagged proteins. The His-tagged proteins adsorb to the Ni-NTA lipids and the monolayer can be transferred to an EM grid that can then be prepared by negative staining or vitrification. To further simplify the technique, we developed Affinity Grids, EM grids with a pre-deposited Ni-NTA lipid monolayer [2]. We have successfully used Affinity Grids to prepare a variety of specimens ranging from soluble proteins and complexes to membrane proteins.

Monolayer purification and Affinity Grids require that the target protein includes a His tag. We are now focusing our efforts on developing a method that is universally applicable and no longer requires the protein of interest to be His-tagged. The easiest way to accomplish this goal is by using His-tagged adaptor molecules. The most general adaptor molecule is protein A, which can recruit proteins or complexes to Affinity Grids as long as a specific antibody against a target protein or a complex constituent is available. We tested the protein A/antibody strategy by isolating ribosomal complexes of native, Flag- or Myc-tagged varieties from mammalian (293T) cell extracts. We also isolated native, untagged human RNA polymerase II (RNAP) from the same cell extract using antibodies against the flexible C-terminal domain linker region. Cryo-EM images of vitrified specimens revealed that many RNAPs were associated with strands of nucleic acids. We were thus able to isolate actively transcribing RNAPs.

Affinity Grids in conjunction with protein A and antibodies are now a universal tool to rapidly produce ice-embedded specimens of pure, functional complexes directly from cell extracts. In combination with tagged protein libraries, the Affinity Grid should prove valuable for highthroughput specimen preparation of macromolecular complexes by single-particle EM.

\section{References}

[1] Kelly, D.F. et al., Monolayer purification: a rapid method for isolating protein complexes for single-particle Electron Microscopy. Proc. Natl. Acad. Sci. U.S.A. 105 (2008) 4703 - 4708.

[2] Kelly, D.F. et al., The Affiinity Grid: a pre-fabricated EM grid for monolayer purification. $J$. Mol. Biol., 382 (2008), 423 - 433. 